\title{
Comment on "Associations between the incidence of antiphosphatidylserine and antiphosphatidylethanolamine antibodies and clinical manifestations of systemic lupus erythematosus"
}

To the Editor We read with great interest an article by Butkiewicz et al. ${ }^{1}$ reporting associations between the presence of antiphosphatidyloserine antibodies (aPS) and antiphosphatidyloethanolamine antibodies (aPE) and the clinical presentation of systemic lupus erythematosus (SLE). The authors studied 71 patients with SLE in comparison with 36 volunteers without autoimmune diseases. To determine the levels of aPS and aPE immunoglobulin (Ig) G and IgM classes, the authors used commercially available double-antibody enzyme-linked immunosorbent assays by DEMEDITEC Diagnostics GmbH, Kiel-Wellsee, Germany, and The Binding Site Inc., San Diego, United States, respectively. The serum concentrations of aPS/aPE of $10 \mathrm{IU} / \mathrm{ml}$ or higher were considered positive. Data on clinical manifestations of SLE and treatment for all patients were obtained from medical records reviewed from 1990 until 2010. The presence of antinuclear antibodies, anticardiolipin antibodies (aCL), lupus anticoagulant (LAC), antiprothrombin antibodies, and anti- $\beta_{2}$ glycoprotein I (anti- $\beta_{2}$ GPI) antibodies was also verified retrospectively. As for clinical symptoms, the authors considered the descriptors of the Systemic Erythematosus Disease Activity Index (SLEDAI) and many other manifestations. Moreover, in all patients, erythrocyte sedimentation rate, $\mathrm{C}$-reactive protein, complete blood count, and echocardiography were assessed.

There were no significant differences in the demographic characteristics between the groups. Of note, the prevalence of hypertension was higher in patients compared with controls $(43.7 \%$ vs $5.0 \%)$. Secondary antiphospholipid syndrome (APS) was present only in $38 \%$ of patients with SLE. Positive aPE were more prevalent in the study group than positive aPS, whereas in controls, only aPE in the IgM class were detected although less frequently than in patients. aPS were detected more frequently in APS patients, but the prevalence of aPE was higher among patients without concomitant APS. The presence of aPS and $\mathrm{APE}$ was generally not related to the presence of other types of autoantibodies. The authors observed myocardial infarction, Raynaud phenomenon, and thrombocytopenia more often in subjects with positive aPS in the IgM class. Moreover, the elevated levels of aPE in the IgM class were associated with a decreased risk of proteinuria.

The data on the prevalence of aPS and aPE among patients with SLE and APS presented by Butkiewicz et al. ${ }^{1}$ are consistent with previous studies; however, the authors did not confirm some of the associations found by other investigators. They refer to the paper by Sciascia et al. ${ }^{2}$ claiming that aPS were included in the set of aPL used in the global anti-phospholipid syndrome score used to validate the risk of thrombosis and pregnancy loss in SLE patients. However, Sciascia et al. ${ }^{2}$ included the antibodies directed against antiphosphatidylserine-prothrombin complex (aPS/PT) and not aPS.

The issue raised by Butkiewicz et al. ${ }^{1}$ may contribute to the discussion on the so called seronegative APS, wherein the clinical symptoms are highly suggestive of APS but consensus antibodies are persistently negative. We also emphasized the usefulness of determining nonconventional aPL in patients with autoimmune diseases when APS is suspected. ${ }^{3}$ It has been shown in other studies that aCL crossreact with other antibodies to negatively charged phospholipids and are unlikely to become independent markers of APS. Butkiewicz et al. ${ }^{1}$ also mention the coexistence of aPS with aCL in patients with secondary APS. Sciascia et al. ${ }^{4}$ reported the diagnostic accuracy of aPS/PT for APS as a whole and for both thrombosis and pregnancy loss. They concluded that adding aPS/PT to LAC and anti- $\beta_{2}$ GPI improves the 
diagnostic power and helps better stratify the risk of thrombosis and pregnancy morbidity. Thus, aPS/PT have been included, among other variables, into 2 scoring systems: the aPL score and the global anti-phospholipid syndrome score.

Butkiewicz et al. ${ }^{1}$ did not confirm a higher incidence of thromboembolic events in aPS-positive subjects. However, the presence of aPS in the IgM class increased the risk of myocardial infarction, with no effect on the occurrence of ischemic heart disease. They do not report any associations between the studied antibodies and pregnancy morbidity. It would be interesting to know the incidence of the latter manifestation of APS in their study group. In other studies some associations were found between aPS, especially of the IgG class, and pregnancy loss. ${ }^{5}$ Finally, the authors did not report any significant associations between $\mathrm{aPE}$ and clinical manifestations of SLE, and those observed for aPS were weak and probably did not have any significant impact on the course of SLE. It might be interesting to study the associations between other antibodies such as the aPS/PT complex, antiphosphatidylinositol, antiphosphatidic acid, or antiprothrombin and clinical symptoms of SLE in a larger group of patients.

Author names and affiliations Magdalena Celińska-Löwenhoff, Teresa Iwaniec, Jacek Musiał (II Department of Medicine, Jagiellonian University Medical College, Kraków, Poland)

Corresponding author Magdalena Celińska-Löwenhoff, MD, PhD, II Katedra Chorób Wewnętrznych, Uniwersytet Jagielloński, Collegium Medicum, ul. Skawińska 8, 31-066 Kraków, Poland, phone: +48-12-430-52-66, fax: +48-12-430-50-68, e-mail: magdalena.lowenhoff@gmail.com

Conflict of interest The authors declare no conflict of interest.

\section{REFERENCES}

1 Butkiewicz F, Kaszuba M, Brzeziński M, et. al. Associations between the incidence of antiphosphatidylserine and antiphosphatidylethanolamine antibodies and clinical manifestations of systemic lupus erythematosus. Pol Arch Med Wewn. 2014; 124: 573-578.

2 Sciascia S, Sanna G, Murru V, et al. GAPSS: the Global Anti-Phospholipid Syndrome Score. Rheumatology (Oxford). 2013; 52: 1397-1403.

3 Musiał J, Swadźba J, Motyl A, et al. Clinical significance of antiphospholipid protein antibodies. Receiver Operating Characteristics plot analysis. J Rheumatol. 2003; 30: 723-730.

4 Sciascia S, Murru V, Sanna G. Clinical accuracy for diagnosis of antiphospholipid syndrome in systemic lupus erythematosus: evaluation of 23 possible combinations of antiphospholipid antibody specificities. J Thromb Haemost. 2012; 10: 2512-2518.

5 Tebo AE. Antiphospholipid syndrome and the relevance of antibodies to negatively charged phospholipids in diagnostic evaluation. Lupus. 2014; 23: 1313-1316. 
